\title{
LncRNA HLA-F-ASI Enhances the Migration, Invasion and Apoptosis of Glioblastoma Cells by Targeting IncRNA MEG3
}

\section{Yanhua Wang \\ Teng Xie \\ Huaming Liu \\ Xiaoping Yu}

Department of Neurosurgery, Hanchuan People's Hospital, Hanchuan City, People's Republic of China
Correspondence: Xiaoping $Y_{u}$

Department of Neurosurgery, Hanchuan People's Hospital, No. I People's Avenue, Hanchuan City, Hubei Province, 431600,

People's Republic of China

Email xiaopingyuavenue@I63.com
Background: Previous studies revealed the oncogenic role of long non-coding RNA (lncRNA) HLA-F-AS1 in colon cancer and breast cancer, while its role in other cancers is unclear. We predicted the direct interaction between HLA-F-AS1 and MEG3, which is a tumor suppressor lncRNA. We then assessed the interaction between HLA-F-AS1 and MEG3 in glioblastoma (GBM).

Methods: The expression levels of HLA-F-AS1 and MEG3 in GBM and paired non-tumor tissues from 60 GBM patients were analyzed by RT-qPCR. Overexpression of HLA-F-AS1 and MEG3 was achieved in GBM cells to explore the interaction between them. The direct interaction between them was confirmed by RNA pull-down assay. The roles of HLA-F-AS1 and MEG3 in cell invasion, migration and apoptosis were explored by Transwell assays and cell apoptosis assay.

Results: HLA-F-AS1 was highly expressed, and MEG3 was downregulated in GBM. Overexpression of HLA-F-AS1 reduced the expression levels of MEG3 while overexpression of MEG3 did not alter the expression of HLA-F-AS1. HLA-F-AS1 increased cell migration and invasion, but decreased cell apoptosis. MEG3 played opposite roles and reduced the effects of HLA-F-AS1 on cell behaviors.

Conclusion: HLA-F-AS1 may sponge MEG3 in GBM cells to promote cell invasion and migration, and to suppress cell apoptosis.

Keywords: glioblastoma, HLA-F-AS1, MEG3, invasion, migration, apoptosis

\section{Introduction}

As the most common type of brain malignancy, glioblastoma (GBM), or grade 4 glioma, is considered as the most lethal cancer. ${ }^{1,2}$ Globally, GBM affects about 3.2 out of 100,000 people. p $^{3,4}$ More than $75 \%$ of these patients will die within a year after the initial diagnosis, and the 10 -year overall survival rate is as low as $5 \%{ }^{5}$ In clinical practices, treatments of GBM mainly include the surgical resection of primary tumors, and chemotherapeutic drugs, radiotherapy and tumor treating field therapy. ${ }^{6-8}$ However, treatment outcomes are generally poor. In effect, no significant improvement in the survival of GBM patients has been made in recent decades. Therefore, novel therapeutic approaches are urgently needed.

The emerging of molecular targeted therapy has providing opportunities to treat advanced cancers, including GBM. ${ }^{9-11}$ With the advantages of high specificity and less adverse effects, targeted therapy can be applied to reverse tumor progression by regulating the expression of cancer-related genes. With the increased elucidation of 
the molecular pathways involved in GBM, certain molecular players, such as ERRFI1 and TACC3, have been characterized as potential targets to treat $\mathrm{GBM}^{12}$ However, targeted therapy for GBM is still under research. Long non-coding RNAs (lncRNAs) are crucial players in cancers. ${ }^{13}$ Elucidating the functions of lncRNAs in cancers provides therapeutic opportunities to treat cancers by targeting lncRNAs. ${ }^{14}$ However, the roles of most lncRNAs in most cancers are unknown. Previous studies revealed the oncogenic role of IncRNA HLA-F-AS1 in colon cancer and breast cancer, and lncRNA HLA-F antisense RNA 1 (HLA-F-AS1) can facilitate the progression of colon cancer and breast cancer. ${ }^{15,16}$ Its role in other cancers should be explored. GBM is closely related to signaling pathways such as tumor necrosis factor (TNF- $\alpha$ ), transforming growth factor- $\beta$ (TGF- $\beta$ ) and signal transducer and activator of transcription $1 / 3$ (STAT1/3). Maternally expressed gene 3 (MEG3) is an imprinted gene that encodes a ncRNA associated with tumorigenesis. It was reported that MEG3 may be an important regulator in the development of glioma. Therefore, we hypothesized that HLAF-AS1 and could interact with MEG3, which is a tumor suppressor. ${ }^{17}$ We then explored the interaction between HLA-F-AS1 and MEG3 in GBM.

\section{Patients, Materials and Methods Patients and Samples}

A total of 62 GBM patients who received surgical resection of the primary tumors at Hanchuan People's Hospital between September 2018 and August 2020 were enrolled as study subjects. Hanchuan People's Hospital Ethics Committee approved this study. All experimental procedures in this study were performed in accordance with the Declaration of Helsinki. Tumors were analyzed by histopathologists. GBM and paired non-tumor tissues were separated from the resected tumors. Tissue samples were kept in liquid nitrogen prior to the subsequent assays. All patients signed the informed consent.

\section{Cells and Transfections}

Two human glioblastoma cell lines U251 and T98G (Cell bank, Chinese Academy of Science) were used. Cells were cultured cultivated in DMEM supplemented with $10 \%$ FBS and $1 \%$ penicillin/streptomycin in an incubator at $37{ }^{\circ} \mathrm{C}$ with $\mathrm{CO}_{2}$ and humidity set to $5 \%$ and $95 \%$, respectively. HLA-F-AS1 and MEG3 were overexpressed in cells by transfecting pcDNA-HLA-F-AS1 or -MEG3 expression vector through transient transfections mediated by Neon Electroporation Transfection (Thermo Fisher Scientific). All operations were performed following the manufacturer's instructions.

\section{RNA Preparation}

High Pure RNA Isolation Kit (Roche Life Science) was used to isolate total RNAs from both tissues and cultivated cells. In each experiment, no less than $10^{7}$ cells or 0.1 g tissue sample were used. The integrity and concentrations of RNA samples were analyzed with Bioanalyzer. In all RNA samples, an RIN value higher than 9.0 were reached, indicating high RNA integrity. RNA concentrations ranged from 900 to $3000 \mathrm{ng} / \mu \mathrm{L}$.

\section{Preparation of cDNA Samples and qPCRs}

The preparation of cDNA samples was carried out using the QuantiNova Reverse Transcription Kit (QIAGEN). Following the protocol, gDNA Removal Mix $(2 \mu \mathrm{L})$, total RNAs $(3 \mu \mathrm{g})$, control RNA and DEPC water were used to prepare a $15 \mu \mathrm{L}$ mixture, which was incubated at $45^{\circ} \mathrm{C}$ for $10 \mathrm{~min}$, followed by incubation on ice for $2 \mathrm{~min}$. After that, reverse transcription enzyme $(1 \mu \mathrm{L})$ and reverse transcription mix $(4 \mu \mathrm{L})$ were added to make a $20 \mu \mathrm{L}$ system, which was then incubated at $25^{\circ} \mathrm{C}$ for $3 \mathrm{~min}, 45^{\circ} \mathrm{C}$ for $20 \mathrm{~min}$, and at $85^{\circ} \mathrm{C}$ for $5 \mathrm{~min}$. qPCR mixtures were prepared with cDNA as the template ( $1 \mu \mathrm{L}$ cDNA sample in a $20 \mu \mathrm{L}$ system) to determine the expression levels of HLA-F-AS1 and MEG3 with $18 \mathrm{~S}$ rRNA as the internal control. The $2^{-\Delta \Delta \mathrm{Ct}}$ method was used to normalize the gene expression levels.

\section{RNA-RNA Pulldown Assay}

A vector expressing HLA-F-AS1 and negative control (NC) RNA with T3 RNA transcriptase was used to prepare RNA transcripts through in vitro transcriptions using the Invitrogen MEGAscript T3 Transcription Kit. Purification of in vitro transcripts was performed using the Invitrogen MEGAclear Transcription Clean-Up Kit. After that, $3^{\prime}$ end labeling with biotin was performed using the BIO $3^{\prime}$-End Oligonucleotide Labeling Kit (Interchim). The labeled RNA transcripts were further purified using the MEGAclear Transcription Clean-Up Kit, followed by transfection into cells through the methods mentioned above. Cells were harvested $48 \mathrm{~h}$ later to perform cell lysis, followed by using Streptavidin agarose beads to isolate RNA complex. Beads were washed to isolate RNA, and RNA samples were subjected to RT-qPCRs to determine the expression levels of MEG3. 


\section{Transwell Assays}

Transwell inserts ( $8 \mu \mathrm{m}$, Corning) were applied to assay the capacities of cells with transfections. Serumfree medium was used to prepare cell suspensions, which were added to the upper chamber. Membrane coated with Matrigel was used in invasion assay. To induce cell movement, the lower chamber was added with $20 \%$ FBS. Cells were cultivated for $24 \mathrm{~h}$. After that, membranes were cleaned, fixed and stained with eosin. Cells were then evaluated under a light microscope.

\section{Cell Apoptosis Assay}

Cells with transfections were further cultivated in serumfree medium for another $48 \mathrm{~h}$. Ice-cold PBS was used to wash cells, and the Annexin V-FITC/PI detection kit (BD Biosciences) was used to stain cells. Cells were incubated in dark for $20 \mathrm{~min}$, followed by flow cytometer (BD Biosciences) to evaluate cell apoptosis.

\section{Statistical Analysis}

The 62 patients were grouped into high and low HLA-F-AS1/ MEG3 groups $(n=31$, median expression level $=$ cutoff value). All experimental data were presented as the Mean \pm SD values. Student's $t$ test was used to compare two groups and One-way ANOVA test was used to compare multiple groups, respectively. The correlation of HLA-F-AS1 and MEG3 was analyzed by linear regression. Associations between the expression levels of HLA-F-AS1 and MEG3 and patients' clinical data such as patients' age, gender and tumor location were analyzed with Chi-squared test. A $p$-value of less than 0.05 was considered statistically significant.

A

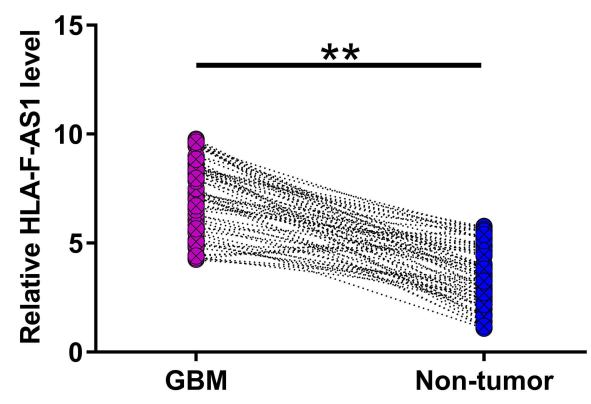

\section{Results}

Differential Expression of HLA-F-ASI and MEG3 in GBM

The differential expression of HLA-F-AS1 and MEG3 in GBM were determined by RT-qPCRs. The results showed that HLA-F-AS1 was highly upregulated (Figure 1A, $p<0.01$ ) and MEG3 was significantly downregulated in GBM (Figure 1B, $p<0.01$ ). Chisquared test showed that the expression levels of HLA-F-AS1/MEG3 were not closely correlated with patients' age, gender and tumor location (Table 1).

\section{The Correlations Between HLA-F-AS I and MEG3}

Pearson's correlation coefficient was carried out to explore the correlations between HLA-F-AS1 and MEG3 across both GBM and non-tumor tissues. It showed that HLAF-AS1 and MEG3 were not closely correlated with each other across GBM (Figure 2A) and non-tumor (Figure 2B) tissues.

\section{The Direct Interaction Between HLA-F-ASI and MEG3}

The interaction between HLA-F-AS1 and MEG3 was first predicted by IntaRNA 2.0 software, followed by validation through RNA-RNA pulldown assay. It was predicted that HLA-F-AS1 and MEG3 could form strong base pairing (Figure 3A). In addition, compared to Bio-NC pulldown group, Bio-HLA-F-AS1 pull-down sample exhibited significantly downregulated MEG3 (Figure $3 \mathrm{~B}, p<0.01$ ), indicating the direct interaction between them.

Figure I Exploration of the differential expression of HLA-F-ASI and MEG3 in GBM. All 62 GBM patients donated GBM and paired non-tumor tissues, which were used to

isolate total RNAs to assay the differential expression of HLA-F-ASI (A) and MEG3 (B) in GBM through RT-qPCRs. **p $<0.0$ I.

B

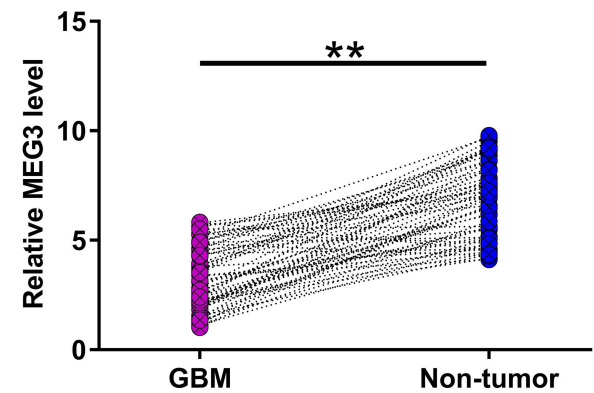


Table I Chi-Squared Analysis of the Associations Between HLA-F-ASI/MEG3 Expression Levels and Patients' Clinical Data

\begin{tabular}{|c|c|c|c|c|c|c|c|}
\hline \multirow[t]{2}{*}{ Variable } & \multirow[t]{2}{*}{ Cases } & \multicolumn{2}{|c|}{ HLA-F-AS I } & \multirow[t]{2}{*}{ P-value } & \multicolumn{2}{|c|}{ MEG3 } & \multirow[t]{2}{*}{ P-value } \\
\hline & & Low & High & & Low & High & \\
\hline \multicolumn{8}{|l|}{ Gender } \\
\hline Male & 40 & 18 & 22 & 0.37 & 21 & 19 & 0.60 \\
\hline Female & 22 & 13 & 9 & & 10 & 12 & \\
\hline \multicolumn{8}{|l|}{ Age } \\
\hline$<45$ & 34 & 18 & 16 & 0.61 & 16 & 18 & 0.61 \\
\hline$\geq 45$ & 28 & 13 & 15 & & 15 & 13 & \\
\hline \multicolumn{8}{|l|}{ Tumor Location } \\
\hline Supratentorial & 44 & 20 & 24 & 0.26 & 21 & 23 & 0.58 \\
\hline Infratentorial & 18 & 11 & 7 & & 10 & 8 & \\
\hline
\end{tabular}

\section{The Role of HLA-F-ASI and MEG3 in the Regulation of Each Other}

Overexpression of HLA-F-AS1 and MEG3 were achieved in U251 and T98G cells. The overexpression was confirmed every $24 \mathrm{~h}$ until $96 \mathrm{~h}$ (Figure 4A, $p<0.05$ ). In both U251 and T98G cells, overexpression of HLA-F-AS1 resulted in downregulation of MEG3 (Figure 4B). However, overexpression of MEG3 showed no effect on the expression of HLA-F-AS1 (Figure 4C).

\section{The Role of HLA-F-ASI and MEG3 in U25I and T98G Cell Behaviors}

Transwell assays and cell apoptosis assay were used to explore the role of HLA-F-AS1 and MEG3 in regulating the behaviors of U251 and T98G cells. HLA-F-AS1 increased cell migration (Figure 5A, $p<0.01$ ) and invasion (Figure 5B, $p<0.01$ ), but decreased cell apoptosis (Figure 5C, $p<0.01$ ). MEG3 played opposite roles and attenuated the effects of HLA-F-AS1 on cell behaviors.

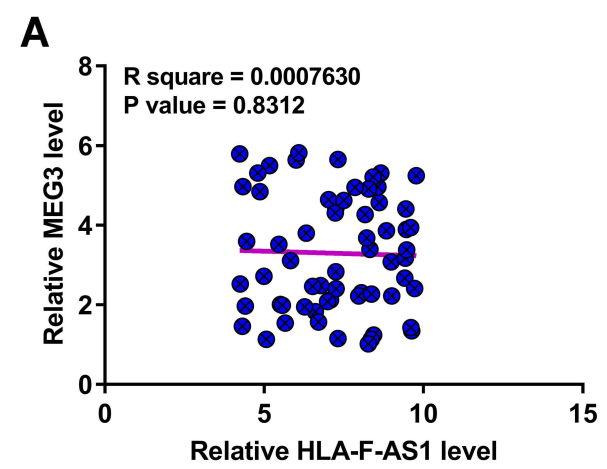

\section{Discussion}

The present study explored the functions of lncRNAs HLA-F-AS1 and MEG3 in GBM, and investigated the potential interaction between them. We observed altered expression of HLA-F-AS1 and MEG3 in GBM. Moreover, HLA-F-AS1 and MEG3 can interact with each other directly, and their interaction suppressed their opposite roles in regulating several behaviors of GBM cells.

Two previous studies characterized the oncogenic role of HLA-F-AS1 in both colorectal cancer and breast cancer. ${ }^{15,16}$ HLA-F-AS1 was upregulated in colorectal cancer, and its overexpression can sponge miR-330-3p to upregulate PFN1, thereby promoting cancer progression. ${ }^{15}$ Breast cancer also exhibits upregulated expression of HLA-F-AS1, which is induced by STAT3. ${ }^{16}$ Overexpression of HLA-F-AS1 increases cancer cell stemness and proliferation by increasing the expression levels of TRABD. The function of HLA-F-AS1 in other types of cancer is unclear. The present study revealed the increased expression levels of HLA-F-AS1. Moreover, overexpression of HLA-F-AS1 resulted in increased cell invasion and migration, as well as reduced cell apoptosis in GBM

B

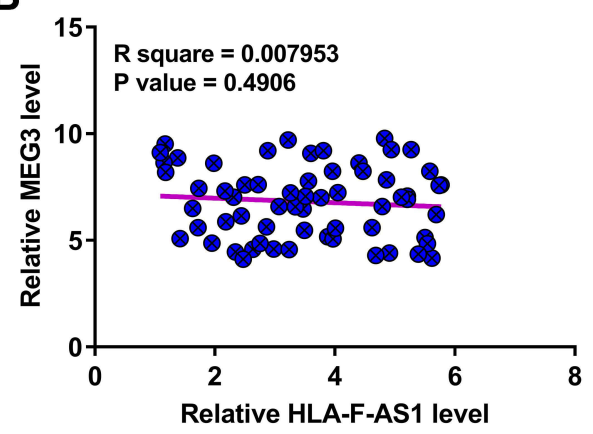

Figure 2 Analysis of the correlations between HLA-F-ASI and MEG3. Pearson's correlation coefficient was carried out to explore the correlations between HLA-F-ASI and MEG3 across both GBM (A) and non-tumor (B) tissues. 
A

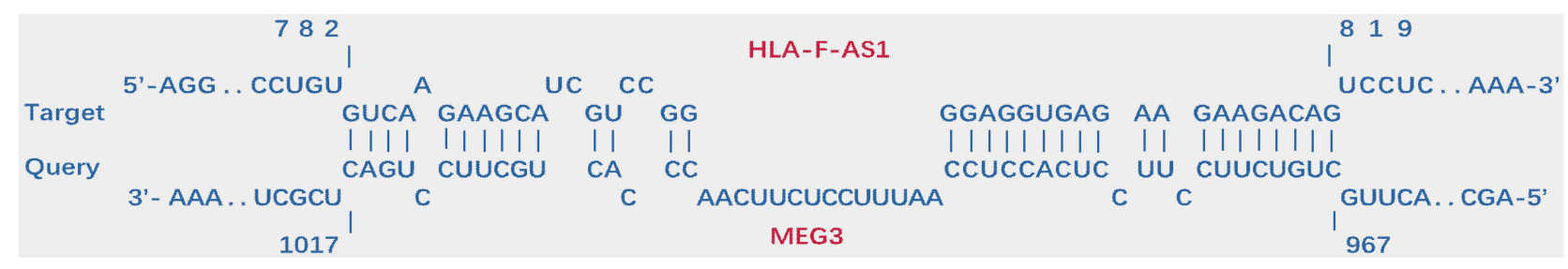

B
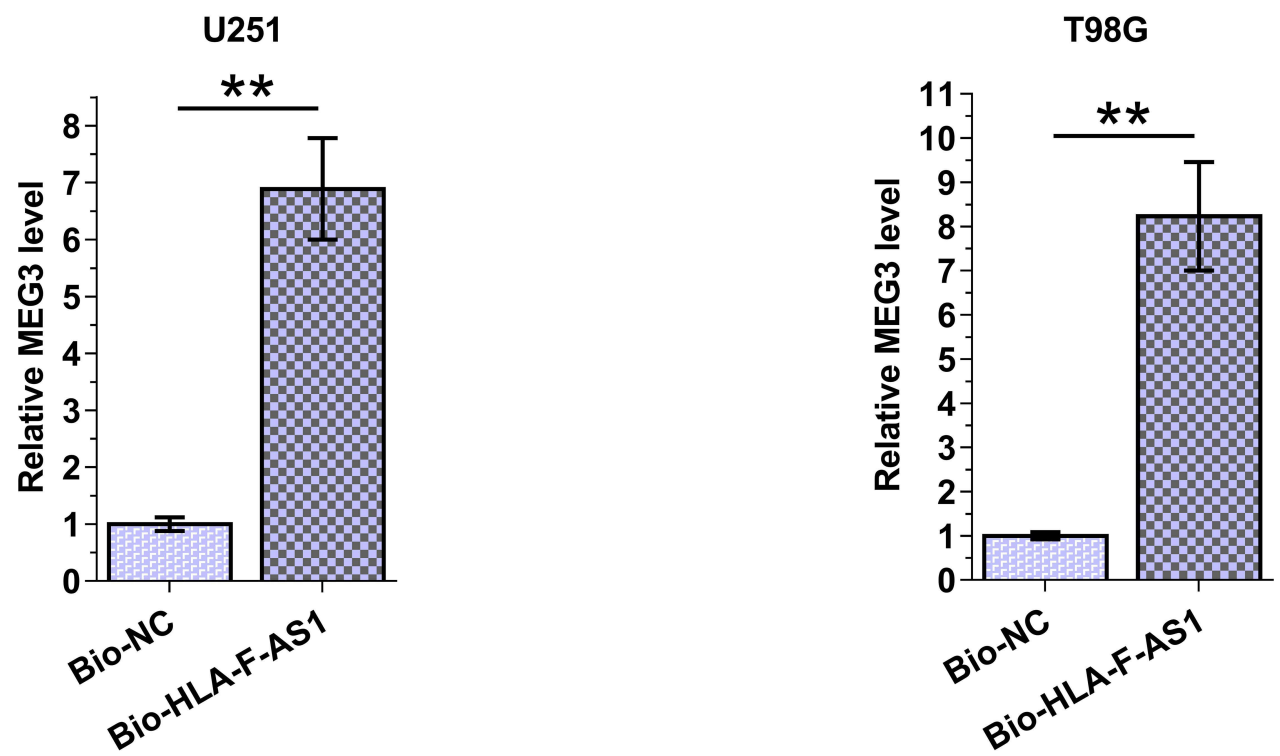

Figure 3 Analysis of the direct interaction between HLA-F-ASI and MEG3. The interaction between HLA-F-ASI and MEG3 was first predicted by IntaRNA 2.0 software (A), followed by validation through RNA-RNA pulldown assay $(\mathbf{B}) .{ }^{* *} p<0.01$.

A

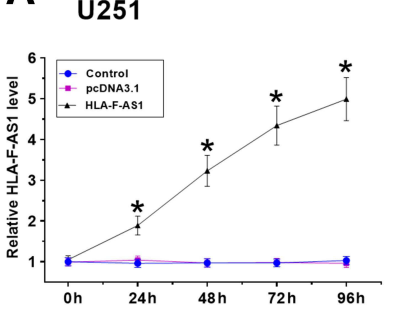

B

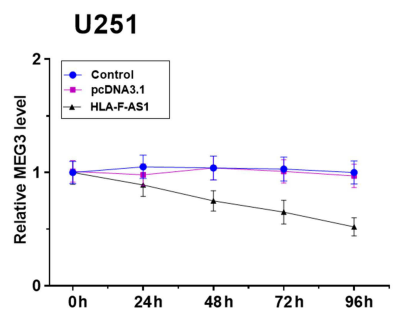

T98G

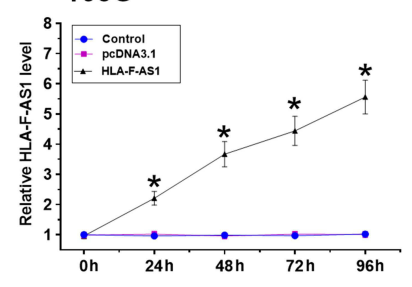

T98G

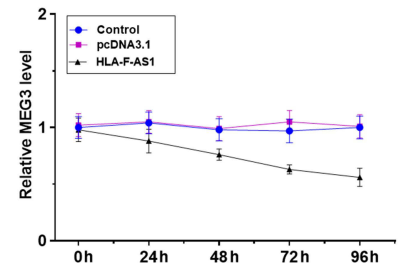

U251

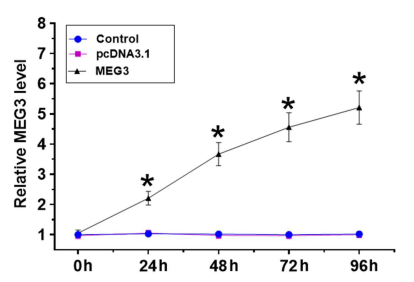

C

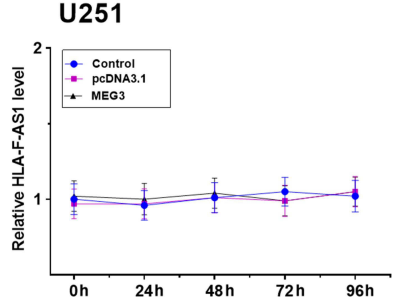

T98G
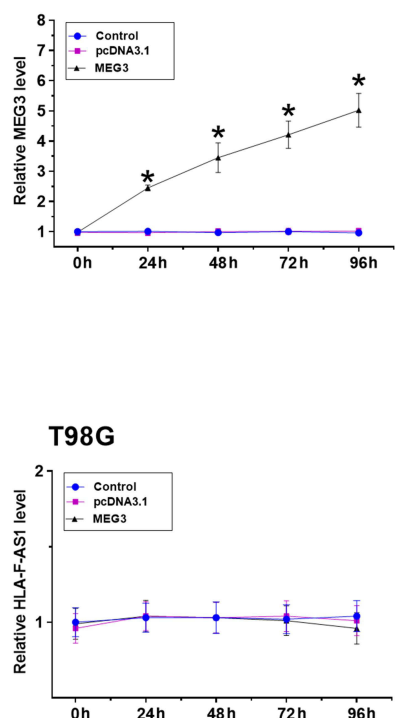

Figure 4 Analysis of the role of HLA-F-ASI and MEG3 in regulating the expression of each other. Overexpression of HLA-F-ASI and MEG3 was achieved in U25I and T98G cells. The overexpression of them was confirmed every $24 \mathrm{~h}$ until $96 \mathrm{~h}(\mathbf{A})$. The role of HLA-F-ASI in regulating the expression of MEG3 (B), and the role of MEG3 in regulating the expression of HLA-F-ASI (C) were analyzed with RT-qPCRs. ${ }^{*} p<0.05$. 

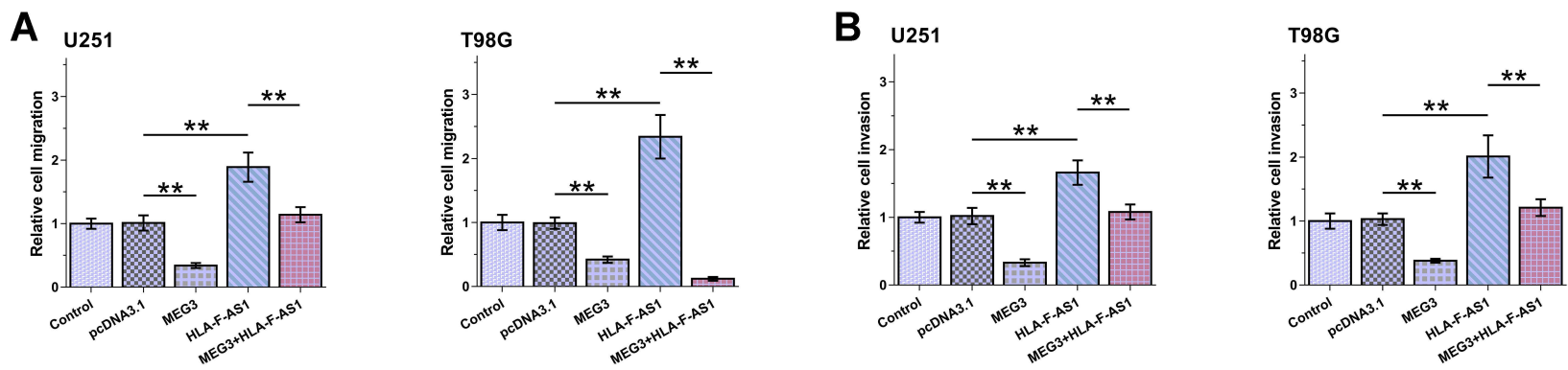

$C_{\text {U251 }}$
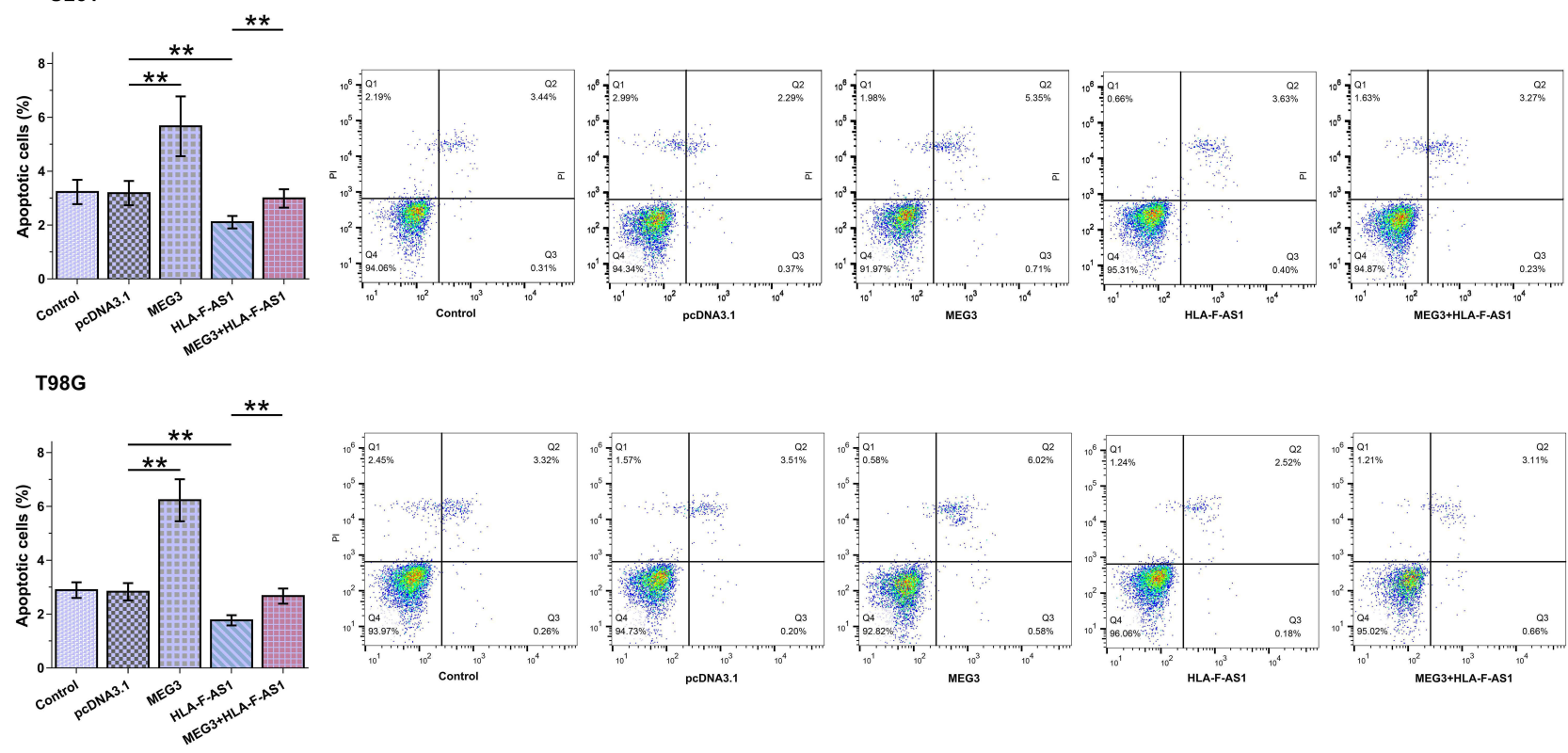

Figure 5 Analysis of the role of HLA-F-ASI and MEG3 in behaviors of U25I and T98G cells. Transwell assays and cell apoptosis assays were used to explore the role of HLA-F-ASI and MEG3 in the migration (A), invasion (B) and apoptosis (C) of U25I and T98G cells. **p $<0.01$.

cells. Therefore, HLA-F-AS1 may promote GBM progression by increasing tumor metastasis and suppressing cancer cell apoptosis.

MEG3 plays tumor suppressive roles in different types of cancer including MEG3. ${ }^{18}$ In GBM, MEG3 suppresses cancer growth and metastasis, and increases the sensitivity of cancer cells to chemical drugs by affecting multiple cancerrelated pathways, such as the PI3K/AKT/mTOR pathway. ${ }^{19}$ The present study confirmed the downregulation of MEG3 in GBM and its role in suppressing tumor metastasis and enhancing cell apoptosis. Based on our knowledge, the regulators of MEG3 in cancer biology remain unclear. In this study, we showed that HLA-F-AS1 and MEG3 could directly interact with each other. However, they showed no role in regulating the expression of each other. However, HLA-F-AS1 suppressed the role of MEG3 in regulating the behaviors of GBM cells. Therefore, we speculated that HLA-F-AS1 could sponge MEG3 to suppress its roles in GBM.
Therefore, the present study reported the interaction between two lncRNAs in GBM, which is novel.

In conclusion, HLA-F-AS1 was upregulated in GBM. HLA-F-AS1 may sponge MEG3 in GBM cells to suppress its tumor suppressive roles in GBM cell metastasis and apoptosis.

\section{Disclosure}

All authors declared no conflicts of interest for this work.

\section{References}

1. Alexander BM, Cloughesy TF. Adult glioblastoma. J Clin Oncol. 2017;35(21):2402-2409. doi:10.1200/JCO.2017.73.0119

2. Holland EC. Glioblastoma multiforme: the terminator. Proc Natl Acad Sci U S A. 2000;97(12):6242-6244. doi:10.1073/pnas.97.12.6242

3. Davis ME. Glioblastoma: overview of disease and treatment. Clin J Oncol Nurs. 2016;20(5 Suppl):S2-8. doi:10.1188/16.CJON.S1.2-8

4. Ostrom QT, Gittleman H, Fulop J, et al. CBTRUS statistical report: primary brain and central nervous system tumors diagnosed in the United States in 2008-2012. Neuro-Oncology. 2015;17(Supp14):iv1iv62. doi:10.1093/neuonc/nov189 
5. Tykocki T, Eltayeb M. Ten-year survival in glioblastoma. A systematic review. J Clin Neurosci. 2018;54:7-13. doi:10.1016/j. jocn.2018.05.002

6. Alifieris C, Trafalis DT. Glioblastoma multiforme: pathogenesis and treatment. Pharmacol Ther. 2015;152:63-82.

7. Zhang H, Wang R, Yu Y, Liu J, Luo T, Fan F. Glioblastoma treatment modalities besides surgery. $J$ Cancer. 2019;10(20):4793-4806. doi: $10.7150 /$ jca. 32475

8. Nam JY, de Groot JF. Treatment of glioblastoma. J Oncol Pract. 2017;13(10):629-638. doi:10.1200/JOP.2017.025536

9. Touat M, Idbaih A, Sanson M, Ligon KL. Glioblastoma targeted therapy: updated approaches from recent biological insights. Ann Oncol. 2017;28(7):1457-1472. doi:10.1093/annonc/mdx106

10. Jain KK. A critical overview of targeted therapies for glioblastoma. Front Oncol. 2018;8:419. doi:10.3389/fonc.2018.00419

11. Le Rhun E, Preusser M, Roth P, et al. Molecular targeted therapy of glioblastoma. Cancer Treat Rev. 2019;80:101896. doi:10.1016/j. ctrv.2019.101896

12. Duncan CG, Killela PJ, Payne CA, et al. Integrated genomic analyses identify ERRFI1 and TACC3 as glioblastoma-targeted genes. Oncotarget. 2010;1(4):265-277. doi:10.18632/oncotarget.137

13. Huarte M. The emerging role of lncRNAs in cancer. Nat Med. 2015;21(11):1253-1261. doi:10.1038/nm.3981
14. Jiang MC, Ni JJ, Cui WY, Wang BY, Zhuo W. Emerging roles of IncRNA in cancer and therapeutic opportunities. Am J Cancer Res. 2019;9(7):1354-1366

15. Huang Y, Sun H, Ma X, et al. HLA-F-AS1/miR-330-3p/PFN1 axis promotes colorectal cancer progression. Life Sci. 2020;254:117180. doi:10.1016/j.lfs.2019.117180

16. Wu D, Jia H, Zhang Z, Li S. STAT3-induced HLA-F-AS1 promotes cell proliferation and stemness characteristics in triple negative breast cancer cells by upregulating TRABD. Bioorg Chem. 2021;109:104722. doi:10.1016/j.bioorg.2021.104722

17. Wang $X$, Li X, Wang Z. IncRNA MEG3 inhibits pituitary tumor development by participating in cell proliferation, apoptosis and EMT processes. Oncol Rep. 2021;45(4). doi:10.3892/or.2021.7991

18. Buccarelli M, Lulli V, Giuliani A, et al. Deregulated expression of the imprinted DLK1-DIO3 region in glioblastoma stemlike cells: tumor suppressor role of lncRNA MEG3. Neuro-Oncology. 2020;22 (12):1771-1784. doi:10.1093/neuonc/noaa127

19. Xu DH, Chi GN, Zhao CH, Li DY. Long noncoding RNA MEG3 inhibits proliferation and migration but induces autophagy by regulation of Sirt7 and PI3K/AKT/mTOR pathway in glioma cells. $J$ Cell Biochem. 2018. doi:10.1002/jcb.28026

\section{Publish your work in this journal}

Cancer Management and Research is an international, peer-reviewed open access journal focusing on cancer research and the optimal use of preventative and integrated treatment interventions to achieve improved outcomes, enhanced survival and quality of life for the cancer patient.
The manuscript management system is completely online and includes a very quick and fair peer-review system, which is all easy to use. Visit http://www.dovepress.com/testimonials.php to read real quotes from published authors. 